\title{
A new method for reducing local heat transfer data in multi-microchannel evaporators
}

\author{
Houxue Huang*, Navid Borhani, Nicolas Lamaison, John R. Thome \\ Laboratory of Heat and Mass Transfer, École Polytechnique Fédérale de Lausanne, \\ EPFL-STI-IGM-LTCM, Station 9, CH-1015 Lausanne, Switzerland
}

\begin{abstract}
Measuring the strong local variation in heat transfer coefficients in multimicrochannel evaporators is related to the inverse heat conduction problem (IHCP). As the local flow heat transfer coefficients change greatly in magnitude from single-phase liquid at the entrance to a peak in slug flow and then to a minimum at the transition to the onset of annular flow and finally a new substantial rise up to the outlet, a significant heat spreading occurs due to the heat transfer process itself, and this has to be accounted for when processing the data. Until now, IHCP has not been introduced in the experimental study of heat transfer in such evaporators when reducing local experimental data. In this paper, a new method for processing experimental local heat transfer data by solving the $3 \mathrm{D}$ IHCP is proposed. This method is then applied and validated using two sets of single- and two-phase flow experimental data obtained with infrared (IR) camera temperature measurements. The 14,400 raw pixel temperatures per image from the IR camera are first
\end{abstract}

\footnotetext{
*Corresponding author: Tel: +41 (0)21 69 36853, Fax: +41 (0)21 6935960

Email address: houxue.huang@epfl.ch; houxue.huang@gmail.com (Houxue Huang)
} 
pre-processed by a filtering technique to remove the noise and then to smooth the data, where the IR camera has undergone a prior inhouse pixel by pixel insitu temperature calibration. Three filtering techniques (Wiener filter, spline smooth, and polynomial surface fitting) are compared. The polynomial surface fitting technique was shown to be more suitable for the current type of data set. Then the 3D IHCP is solved based on a finite volume method using the TDMA (Tridiagonal Matrix Algorithm) solver with a combination of Newton-Raphson iteration and a local energy balance method. Furthermore, the present 3D TDMA method (named as 3D TDMA) is compared with three other post-processing methods currently used in the literature, among which the present one is found to be more accurate for reducing the local heat transfer data in multi-microchannel evaporators.

Keywords: Local heat transfer coefficients, multi-microchannel evaporator, temperature filtering techniques, heat conduction models, inverse heat conduction problem 


\section{Contents}

Introduction $\quad 4$

$\begin{array}{ll}\text { Case descriptions } & 9\end{array}$

Case I: Single-phase flow . . . . . . . . . . . . . . . . . 10

Case II: Two-phase flow . . . . . . . . . . . . . . . . . . . . . 13

$\begin{array}{ll}\text { Theory } & 14\end{array}$

Filtering techniques . . . . . . . . . . . . . . . . . 14

$3 \mathrm{D} \mathrm{IHCP} \ldots \ldots \ldots \ldots$

Boundary conditions . . . . . . . . . . . . . . . . . 18

3D TDMA ..................... 20

Newton-Raphson iteration . . . . . . . . . . . . . . 22

Energy balance method . . . . . . . . . . . . . 23

Current heat conduction models in literature . . . . . . . . . . . 23

1D-Direct model . . . . . . . . . . . . . . . 23

2D-TDMA model . . . . . . . . . . . . . . . 25

3D-Direct model . . . . . . . . . . . . . . . . 26

$\begin{array}{ll}\text { Analysis of results and discussion } & 27\end{array}$

Filtering techniques comparison . . . . . . . . . . . . . . 27

Comparison of heat conduction models . . . . . . . . . . . . 31

Case I: Single-phase flow . . . . . . . . . . . . . . . . . . . . 31

Case II: Two-phase flow . . . . . . . . . . . . . . . . . 35

$\begin{array}{ll}\text { Conclusion } & 38\end{array}$ 


\section{Introduction}

The experiments on flow boiling in multi-microchannel evaporators aim to measure the local heat transfer coefficients at the interface between the wall and the fluid along the flow channels. However, only the heat flux and the temperature at the test section backside can be directly measured, whilst the heat spreading due to the gradient in the local saturation temperature and the significant variation in the heat transfer coefficient along the channels needs to be accounted for. In particular, as the local flow heat transfer coefficients change greatly in magnitude from single-phase liquid at the entrance to a peak in slug flow to a minimum at the transition to annular flow and then a new substantial rise up to the outlet, a significant heat spreading occurs due to the heat transfer process itself, and this has to be accounted for when processing the data. Accurate multi-microchannel evaporator data are of paramount importance to the building and validation of prediction methods used to simulate the local temperatures in a CPU or GPU, which must remain below their operating limit.

In such experiments, normally the heat flux is provided by a DC electrical microheater sputtered on the test section backside. The backside temperature traditionally is measured by thermocouples [1] or resistance temperature detectors (RTDs) [2]. These standard techniques provide the temperature mea- 
surements with a point value or a spatial averaged value, which is regarded to be zero-dimensional in terms of spatial resolution. Such data are usually reduced assuming that only 1D heat conduction occurs to obtain local heat transfer coefficients. Hence this technique falls short when monitoring the temperature information with high spatial variation.

Infrared cameras are able to provide "high definition" temperature measurements and are becoming more widely implemented for microscale heat transfer study [3-8]. Compared to standard techniques, the IR camera tends to be superior due to several merits such as non-intrusive measurements, truely two-dimensional temperature maps, high frequency acquisition, etc. For instance, the non-intrusive technique avoids any interference on the temperature fields, the high resolution temperature map enables one to quantitatively evaluate the axial heat conduction, and the high frequency acquisition allows for transient thermal measurement. A pixel by pixel inhouse calibration also allows the IR temperature accuracy to become equivalent of those of thermocouples.

From the perspective of theoretical heat conduction models, the directly measured temperatures and heat flux on the test section backside are respectively considered to be the Dirichlet and Neumann boundary conditions while the desired heat transfer coefficients on the opposite side (on top) are the Robin boundary condition. The problem of determining the heat transfer coefficients at the top boundary from the temperatures and heat flux measured at the bottom boundary and other boundary conditions by solving the heat conduction equation is regarded as an inverse heat conduction problem (IHCP) [9]. The principal difficulty in solving IHCP is due to its sensitivity 
to noise in the input temperature data. This noise incurs oscillations in the heat flux field since it is magnified while calculating the Laplacian term, or the second derivative of the temperature in the heat conduction equation. To our knowledge, so far two solution strategies have been proposed in the literature to overcome such problems: (1) an iterative regularization scheme [10-14], and (2) a pre-filtering of the raw temperature data [15-17]. The former iterative scheme was first implemented in [10]. This method is based on the least square error between the measured and simulated temperature data. A regularization parameter is involved in the least square formula. A proper selection of this parameter is able to cope with the noise in the input raw temperature data to further alleviate oscillations in the final solution. In this regard, the most general example is the iterative regularization scheme coupled with a conjugate gradient method [18]. However, this method for solving IHCP is only practical for traditional temperature sensors which provide limited numbers of input and output data [9]. Instead, for applications with a large number of input temperatures from an IR camera, this method has shown to be cumbersome due to its expensive computational cost [19]. As an alternative, pre-process filtering of the raw temperature data has proven to be efficient at handling such large noisy data $[15,16]$. The preprocess filtering technique is therefore adopted in our present work. In this second approach, recently three filtering techniques (an ideal low-pass filter, a Gaussian filter, and a Wiener filter) have been compared in [16] with respect to an example of heat source restoration in a thin plate. They found that the consecutive implementation of the Wiener filter yielded the most suitable results. In their work, the $2 \mathrm{D}$ heat conduction model was built assuming 
a constant temperature distribution along the thickness direction due to a very low Biot number $(\ll 1)$. It is worth noting that the filtering technique appears not to be suitable for the case of a limited number of measurement points obtained by thermocouples or RTDs.

Until now only a few researchers have considered the IHCP when reducing their flow boiling heat transfer data in minichannels. Poniewski and coworkers $[20,21]$ have applied IHCP to flow boiling in single small diameter tubes when using liquid crystals to obtain high resolution external temperature fields. Luciani et al. [22] applied the inverse method to reduce the local flow boiling heat transfer data of HEF-7100 in single minichannels under normal, hyper-, and microgravity. The boundary element method was implemented to resolve the inverse heat conduction problem in $2 \mathrm{D}$ and $3 \mathrm{D}$ models to characterize the local wall heat flux and temperature.

So far, the concept of IHCP has not been introduced into the experimental local heat transfer data reduction in multi-microchannel evaporators. In most experimental studies, a 1D heat conduction model was employed to calculate the heat transfer coefficients using the measured temperature data at the backside of the test section without any iteration $[1,4]$. The $1 \mathrm{D}$ model only considers the heat conduction through the thickness direction, assuming no heat spreads in the axial direction, which is only suitable for small spatial temperature variations. The axial heat conduction was evaluated in a non-uniform heat flux study using diode temperature sensors by the local 2D energy balance method [23]. That allowed a better determination of the net local base heat flux than a 1D approach. However, a 1D heat conduciton model was still used to conduct the calculation up to the wall temperature 
using this adjusted base heat flux. Also, a full 2D model was implemented by Costa-Patry and Thome [2] using an array of 35 silicon diode temperature sensors to study flow boiling of refrigerants in a multi-microchannel evaporator with a non-uniform heat flux, i.e. hot spot(s) in the imposed heat flux. However, they only considered the $2 \mathrm{D}$ heat condution from the bottom to the top without any iteration. This is referred from now on as the "direct" method. Recently, a 3D heat conduction model was solved directly from observations at the back of a multi-microchannel test section to characterize the local heat transfer coefficients, without any iteration or resorting to IHCP $[8]$.

In order to implement and validate the $2 \mathrm{D}$ conduction model used in their dynamic modeling of multi-microchannel evaporators [24], the authors introduced an iterative approach to solve the $2 \mathrm{D}$ heat conduction problem by using a guessed heat transfer coefficient profile at the top boundary and the known base temperature profile as the investigator [25]. However, three shortcomings remained in their work: (1) the heat transfered in the widthwise direction (perpendicular to the flow) was ignored (i.e. 2D only), (2) the local fluid temperature is needed, (3) the iteration with the guessed Robin boundary condition at the top appears to be much more time-consuming than the direct method of [8].

In the present work, we aim to provide a more practical and precise data processing method by solving the 3D IHCP for obtaining the local heat transfer data in multi-microchannels (single- and two-phase cases). This method is validated using two sets of single- and two-phase flow experimental data tests for a multi-microchannel test section. In the experiment, the 
IR camera was employed as the temperature sensor. Due to the high resolution of the IR temperature map, an improved way to determine the lateral boundary conditions is introduced. To remove the noise in the raw temperature data, three filtering techniques are employed: Wiener filtering, spline smoothing, and polynomial surface fitting. In the new method, the direct 3D heat conduction problem is solved firstly using a finite volume method (TDMA algorithm) with a guessed Dirichlet boundary condition at the top (instead of the time-consuming Robin boundary condition of [25]). Then a Newton-Raphson method is used to optimize the new footprint temperature array. Finally, the local energy balance method is employed to determine the local heat flux at the top boundary. As a final test, three heat conduction models used in the current literature to reduce the experimental data are reviewed and compared with the new proposed method to demonstrate why it is important to go to this much detail to get accurate local heat transfer results.

\section{Case descriptions}

Two cases of experimental data composed of single- and two-phase flow were selected to validate the new method. The experimental facility used to acquire the data was the same as presented in [8], in which the details of the experimental setup and operation procedures were also introduced. In this part, only the experimental conditions are highlighted. As shown in Fig. 1 , the microchannel evaporator tested during this experiment is made from silicon with 67 parallel microchannels, each $100 \times 100 \mu \mathrm{m}^{2}$, with an inlet restriction ratio of 2 to stabilize the two-phase flow and obtain good flow 
distribution. The channels are $10 \mathrm{~mm}$ long and the test section is $10 \mathrm{~mm}$ wide. The low pressure refrigerant R245fa was used as the test fluid. The uniform heat flux was provided by two micro-heaters sputtered on the back side of the test section with a thickness of $1.5 \mu \mathrm{m}$. The temperature map at the test section bottom covered by a high-emissivity matt tape was acquired by an IR camera (FLIR ThermaCAM SC3000) coupled with a close up lens (LW 34/80) at a frequency of $60 \mathrm{~Hz}$ and a resolution of $320 \times 240$ pixels. The IR camera was calibrated inhouse pixel by pixel (see [8] for calibration details) to an accuracy of $0.2 \mathrm{~K}$ according to the error propagation procedure, although at an isotermal condition all the pixels agreed within a band of $\pm 0.2 \mathrm{~K}$. The pixels corresponding to the test section area of $10 \times 10 \mathrm{~mm}^{2}$ gave an array of $120 \times 120$ pixels (14,400 temperatures per image), leading to a fine-resolution for the local footprint heat flux $q_{f t p}$ and temperature $T_{f t p}$. This high spatial resolution however exaggerates the impact of the signal noise on the solution when solving the heat conduction equations. As a result, the original IR temperature signals need to be pre-processed by a filtering technique.

Case I: Single-phase flow

During the single-phase flow test, the saturation temperature was set to $60^{\circ} \mathrm{C}$, allowing the subcooled fluid temperature to have enough room to increase without reaching saturation or subcooled boiling. The single-phase flow test was carried out at a mass flux of $G=2000 \mathrm{~kg} \mathrm{~m}^{-2} \mathrm{~s}^{-1}$ and a heat flux of $q=20 \mathrm{~W} \mathrm{~cm}^{-2}$ with the inlet fluid temperature of $27.4{ }^{\circ} \mathrm{C}$. The energy loss in this test to the housing of the test section and the ambient was quantified as $20.5 \%$ (based on a single-phase flow energy balance using the 


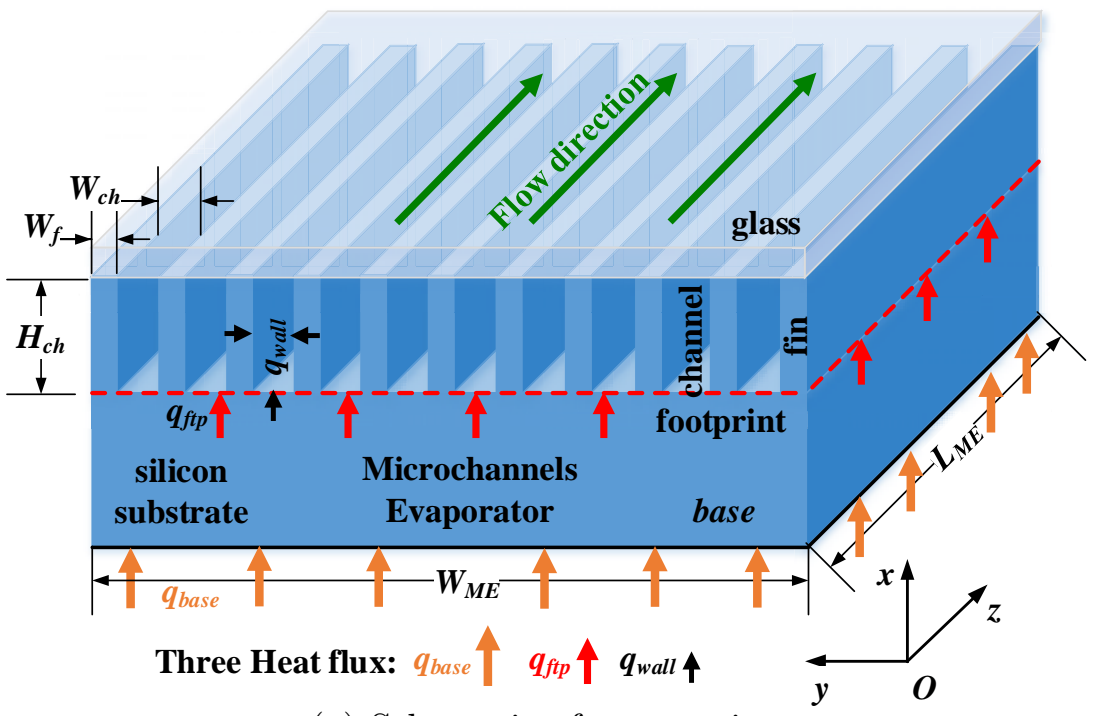

(a) Schematic of test section

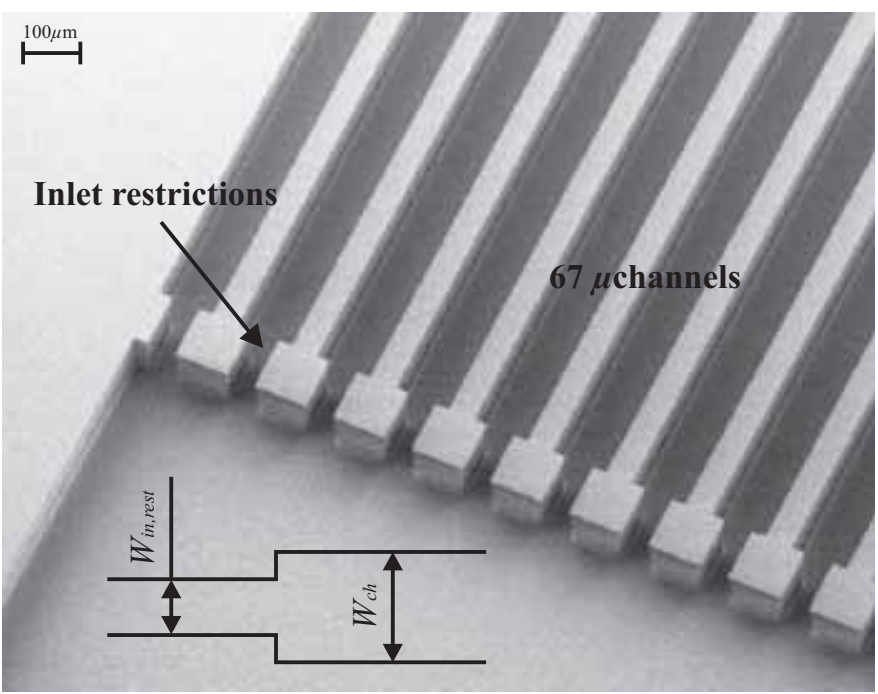

(b) Photo of microchannels with inlet orifices [8]

Fig. 1: Schematic of the multi-microchannel evaporator and a photo of the microchannels with inlet orifices. 
mass flow rate and the measured inlet and outlet temperatures). The local fluid temperature can be evaluated with the local $q_{f t p}$ obtained from the heat conduction model presented in the latter section. The local fluid temperature $T_{f l}$ can then be calculated locally based on the local energy balance:

$$
q_{f t p}(y, z)=c_{p} \dot{m} \Delta T(y, z)
$$

The local footprint heat transfer coefficients are calculated using the local values of $q_{f t p}, T_{f t p}$ and $T_{f l}$, and the wall heat transfer coefficients are attained using the fin efficiency equations:

$$
\begin{aligned}
& \alpha_{f t p}(y, z)=\frac{q_{f t p}(y, z)}{T_{f t p}(y, z)-T_{f l}(y, z)} \\
& \alpha_{w a l l}(y, z)=\frac{\alpha_{f t p}(y, z) W_{M E} L_{M E}}{N_{c h}\left[W_{c h} L_{M E}+2\left(L_{M E}+W_{f} / 2\right) H_{c h} \eta_{f}(y, z)\right]} \\
& \eta_{f}(y, z)=\frac{\tanh \left[m(y, z) H_{c h}\right]}{m(y, z) H_{c h}} \\
& m(y, z)=\sqrt{\frac{2 \alpha_{w a l l}(y, z)\left(L_{M E}+W_{f}\right)}{k_{S i}(y, z)\left(L_{M E} W_{f}\right)}}
\end{aligned}
$$

Since the local fin efficiency is unknown, Eqs. (3) to (5) need to be solved iteratively with an initially assumed fin efficiency until the convergence criterion is met. Finally, the local Nusselt number is calculated using:

$$
N u(y, z)=\frac{\alpha_{\text {wall }}(y, z) D_{h}}{k_{f l}(y, z)}
$$


Case II: Two-phase flow

For the two-phase flow test, the outlet saturation temperature was set at $31.5^{\circ} \mathrm{C}$. The two-phase experiment was conducted under a mass flux of $G$ $=2000 \mathrm{~kg} \mathrm{~m}^{-2} \mathrm{~s}^{-1}$ and a heat flux of $q=20 \mathrm{~W} \mathrm{~cm}^{-2}$ with the subcooled inlet fluid temperature at $30.4^{\circ} \mathrm{C}$ and an inlet subcooling of $5.4 \mathrm{~K}$. The energy loss in this test was evaluated as 14\%. Contrary to the single-phase flow test, the local fluid temperature in two-phase flow cannot be obtained based on an equation such as Eq. (1) since the flow is evaporating and the local fluid temperature is directly linked to the local pressure. The latter is generally predicted assuming a linear variation or using a two-phase pressure drop correlation. Therefore, the two-phase flow section will not exhibit any $\alpha_{\text {wall }}$ calculations (for which the fluid temperature profile is needed) but will rather end up with $q_{f t p}$ and $T_{f t p}$ calculations from the heat conduction model proposed below.

The uncertainty analysis based on the approach proposed by Kline and McClintock [26] and the resulted uncertainty propagation were performed in this study. The obtained uncertainties of the main parameters are presented in Table 1. The footprint heat flux and temperature $\left(T_{f t p}\right.$ and $\left.q_{f t p}\right)$ were obtained by solving the 3D IHCP numercially. Their numerical uncertainties caused by the input measurements uncertainty $\left(T_{I R}\right.$ and $\left.q_{b a s e}\right)$ was evaluated by the "Brute force method" [27]. 
Table 1: Experimental measurement uncertainties of main parameters.

\begin{tabular}{lccc}
\hline Parameter & Unit & \multicolumn{2}{c}{ Uncertainty } \\
& & $\mathrm{SP}$ & $\mathrm{TP}$ \\
\hline$T_{t c}$ & ${ }^{\circ} \mathrm{C}$ & $\pm 0.1{ }^{\circ} \mathrm{C}$ & \\
$T_{I R}$ & ${ }^{\circ} \mathrm{C}$ & $\pm 0.2{ }^{\circ} \mathrm{C}$ & \\
$q$ & $\mathrm{~W} \mathrm{~cm}^{-2}$ & $\pm 0.16 \%$ & \\
$G$ & $\mathrm{~kg} \mathrm{~m}^{-2} \mathrm{~S}^{-1}$ & $\pm 4.25 \%$ & \\
$T_{f t p}$ & ${ }^{\circ} \mathrm{C}$ & $\pm 0.44 \%$ & $\pm 0.49 \%$ \\
$q_{f t p}$ & $\mathrm{~kW} \mathrm{~m}^{-2}$ & $\pm 5.9 \%$ & $\pm 11.6 \%$ \\
$\alpha_{f t p}$ & $\mathrm{~kW} \mathrm{~m}^{-2} \mathrm{~K}^{-1}$ & $\pm 5.7 \%$ & $\pm 12.1 \%$ \\
$\alpha_{\text {wall }}$ & $\mathrm{kW} \mathrm{m}{ }^{-2} \mathrm{~K}^{-1}$ & $\pm 6.3 \%$ & $\pm 8.3 \%$ \\
$N u$ & - & $\pm 9.4 \%$ & \\
\hline & & &
\end{tabular}

\section{Theory}

\section{Filtering techniques}

Three filtering techniques were considered and compared here. They are discussed below.

Wiener filter. The Wiener filter has been shown to be an efficient tool to remove the noise in the raw data of ill-posed IHCP [16, 28]. The principle advantage of this filtering technique is related to its self-adaptivity by tailoring itself to the local noise level. Based on a statistical approach, the Wiener filter estimates the new data pixel by pixel from its neighborhood data points by minimizing the square of the error between the estimated data and the original one. The local mean and variance of each data point are defined as 
follows:

$$
\begin{aligned}
\mu & =\frac{1}{E \cdot F} \sum_{i, j \in \Omega} T_{e x p}(i, j) \\
\sigma^{2} & =\frac{1}{E \cdot F} \sum_{i, j \in \Omega}\left[T_{e x p}(i, j)-\mu\right]^{2}
\end{aligned}
$$

where $\Omega$, the local neighborhood, consists an array of E-by-F pixels. Then the filtered sample temperature can be estimated as:

$$
\tilde{T}_{e x p}(i, j)=\mu+\frac{\sigma^{2}-v^{2}}{\sigma^{2}}\left[T_{e x p}(i, j)-\mu\right]
$$

where $v$ is the noise variance (assumed as additive Gaussian white noise if it is unknown). This technique requires an adjustment to filter the data points close to the boundary. To overcome this problem at the boundary of the pixel field, a different window size can be used to make the statistical estimation.

Spline smoothing. The spline function is another efficient tool to smooth noisy or discrete data [29]. The two-dimensional bicubic spline $s(x, y)$ is expressed in its B-spline series representation [30] as:

$$
s(x, y)=\sum_{i=1}^{p} \sum_{i=1}^{r} C_{i j} M_{i}(x) N_{j}(y)
$$

where $M_{i}(x)$ and $N_{j}(y)$ are the normalized cubic B-splines, and the coefficients $C_{i j}$ are determined by solving the following constrained minimization problem: Minimize $\gamma$ with the constraint $\theta_{r} \leq S$, where $\gamma$ is a measure of the lack of smoothness of $s(x, y), \theta_{r}$ represents the weighted residual $\omega_{r}\left[f_{r}-s(x, y)\right]$, and $S$ controls the extent of smoothing and is thus also named 
the smoothing factor [31].

Polynomial surface fitting. Fitting data from a surface using polynomials has been used for geophysical data [30]. The value at each calculated point is determined by implementing a polynomial surface fit to the neighboring data with a least square approach:

$$
\begin{array}{r}
\tilde{T}(x, y)=\sum_{i=0}^{N} \sum_{j=0}^{N-i} A_{i j} x^{i} y^{j} \\
R=T(x, y)-\tilde{T}(x, y)
\end{array}
$$

where $N$ is the polynomial degree, $A_{i j}$ are the polynomial coefficients, and $x, y$ denote the location of the point. Once the polynomial degree is determined, the polynomial coefficients are identified by solving the minimization problem, $\sum R^{2}=$ minimum, using least square method.

\section{D IHCP}

Turning now to the steady state 3D heat conduction problem, the present experimental case is governed by the following expressions:

$$
\begin{aligned}
\frac{\partial}{\partial x}\left(k \frac{\partial T}{\partial x}\right)+\frac{\partial}{\partial y}\left(k \frac{\partial T}{\partial y}\right)+\frac{\partial}{\partial z}\left(k \frac{\partial T}{\partial z}\right) & =0 \\
T(0, y, z)=T_{I R}, k \frac{\partial T}{\partial x}(0, y, z) & =q_{b} \\
k \frac{\partial T}{\partial y}(x, 0, z)=q_{s}, k \frac{\partial T}{\partial y}\left(x, W_{M E}, z\right) & =q_{n} \\
k \frac{\partial T}{\partial z}(x, y, 0)=q_{w}, k \frac{\partial T}{\partial z}\left(x, y, L_{M E}\right) & =q_{e}
\end{aligned}
$$


where $x, y, z$ indicate the direction in the thickness $x$ and the widthwise $y$ and lengthwise $z$ (flow) directions, as shown in Fig. 2, and $q_{w}, q_{e}, q_{n}$ and $q_{s}$ are the heat fluxes at the four lateral sides of the substrate. The thickness of the micro-heater $(1.5 \mu \mathrm{m})$ was neglected. Currently, in most of the literature, the heat fluxes at the lateral sides are assumed to be zero (adiabatic). Due to this assumption, the local footprint heat flux at the perimeter of the evaporator would be obviously higher than in reality. This is because the heat actually lost by conduction to the surrounding base of the test section inadvertently then gets assigned to the nodes near and at the perimeter while the measured temperatures in those pixels are lower. To take this into account, a new way to approximately determine the Neumann boundary condition at the four lateral sides are proposed based on the IR temperature map. Here the Neumann boundary condition at each of the four lateral sides is assumed to be constant, and its calculation is presented in next section.

Additionally, instead of the regular way for solving IHCP using an itera-

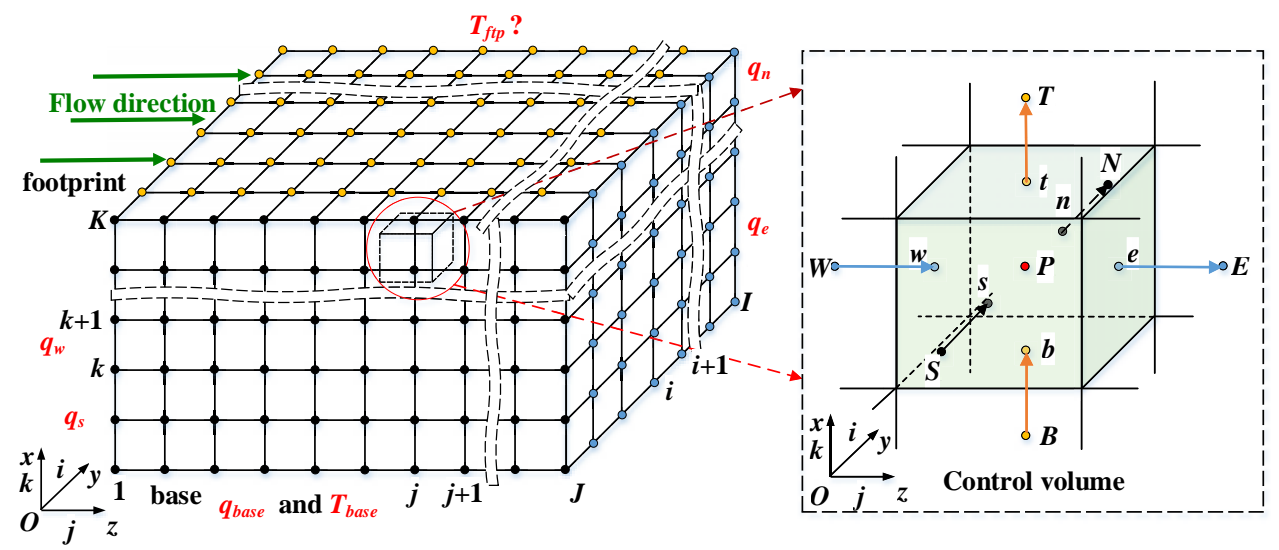

Fig. 2: Mesh types used in the 3D-TDMA model. 
tive regularization scheme, as mentioned previously, a new method based on a temperature filtering technique is used. The main steps used in the new method to solve the 3D IHCP are as follows:

(1) Filter the raw IR temperature data;

(2) Solve the direct 3D heat conduction problem based on the finite volume method using a 3D TDMA solver with an initial guessed temperature map at the footprint $T_{f t p}^{k}$ (i.e. top surface in Fig. 2);

(3) Check if $\delta T_{\text {base }}=T_{\text {base,cal }}-T_{\text {base,meas }} \leq \epsilon$ to decide whether to stop or continue ( $\epsilon$ being the convergence criterion);

(4) Use the single variable Newton-Raphson method locally to optimize the new temperature map at the footprint: $T_{f t p}^{k+1}=T_{f t p}^{k}-\frac{\delta T_{\text {base }}}{T_{\text {base }_{\text {pert }}}}$;

(5) Proceed back to step 2 with $T_{f t p}^{k+1}$ until the convergence criterion is achieved;

(6) Implement the local energy balance at the footprint to determine the local heat flux, $q_{f t p}$.

The flow chart used in the new method is depicted in Fig. 3.

\section{Boundary conditions}

The Neumann boundary condition at the lateral sides refer to $q_{w}, q_{e}, q_{n}$ and $q_{s}$ in the 3D model, as shown in Fig. 2. So far in the open literature, these boundary conditions have been traditionally assumed to be adiabatic, i.e. no heat loss at the lateral sides. This assumption is mainly due to the fact that there is no reference related to the boundary condition from the traditional experimental perspective. However, it is far from reality to ignore the heat conduction from the heated silicon test section to the surrounding thickness of the base of the finned cold plate evaporator and its manifold, especially for 


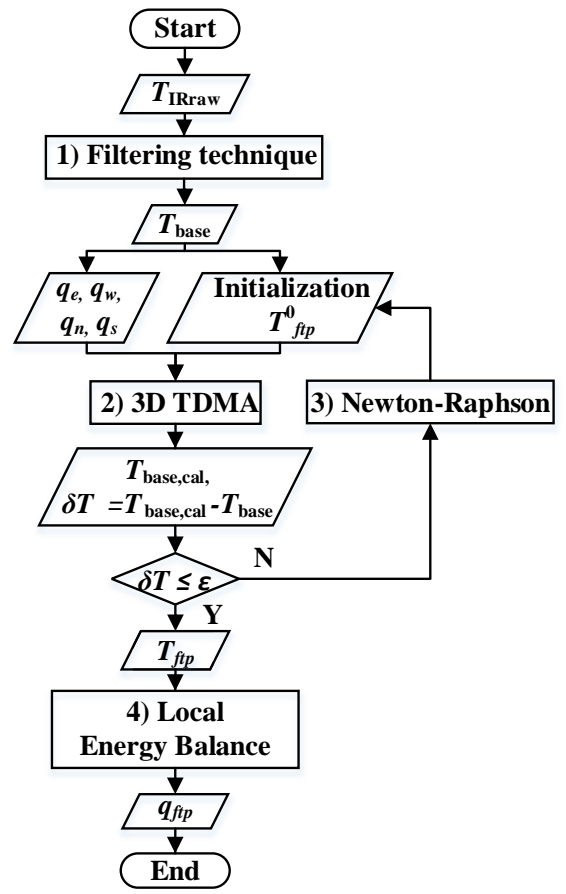

Fig. 3: Flow chart of the new method.

those made of copper on silicon. Thanks to the fine-resolution temperature map from the IR camera, approximate Neumann boundary conditions can be defined.

Applying the definition of the heat flux $q=-k \frac{d T}{d z}$ at the boundary, it is simple to calculate the heat flux if the temperature gradient $\frac{d T}{d z}$ is known. In this regard, the $2 \mathrm{D}$ temperature map from the IR camera provides sufficient information. The total lateral heat conduction $Q_{\text {lateral }}$ leaving the test section through its sides is thus evaluated by summarizing the heat loss at the four sides. Then, in order to respect the energy balance, the base heat flux $q_{b}$ can be adjusted from the total heat power $Q_{\text {total }}$ by combining the total heat loss 
and the lateral heat conduction as follows:

$$
\begin{aligned}
& q_{b} A_{b}=Q_{\text {total }}-\left(Q_{\text {loss }}-Q_{\text {lateral }}\right) \\
& Q_{\text {lateral }}=q_{w} A_{w}+q_{e} A_{e}+q_{s} A_{s}+q_{n} A_{n}
\end{aligned}
$$

where $w, e, s$ and $n$ refer to the four directions. The above equations essentially redistribute the heat loss in a more rational way, thus allowing the base heat flux to be more accurately evaluated by accounting for the lateral heat loss in the heat conduction model. In this manner, the pixels influenced by the lateral heat conduction around the perimeter of the test section can be quantitatively identified and thus removed from the heat transfer database.

\section{D TDMA}

The 3D heat conduction equation is a non-linear elliptical equation. Therefore, the solution is impacted by all of the boundary conditions. This means that the boundary conditions at all six boundaries should be simultaneously solved in the calculation. One might note that the boundary condition at the footprint is missing. However, there are two boundary conditions at the base, a Dirichlet and a Neumann boundary condition. Therefore, the 3D heat equation can be solved with the footprint temperature as the objective and the measured base (bottom) temperature the investigator, i.e. iterating on the $T_{f t p}$ boundary condition in order to satisfy the condition on the measured base temperature (as already shown in Fig. 2).

The 3D heat conduction equation was discretized based on the finite volume method, which integrates the equation on a control volume basis [32]. One advantage of this method is to monitor the local energy balance at 
each control volume. The vertex-centered grid type was implemented in the discretization of the silicon substrate, as demonstrated in Fig. 2, because this grid type allows the direct use of the base temperature map as the investigator. In order to maintain the energy balance at the interface of two neighboring control volumes, the harmonic average thermal conductivity between the two neighboring nodes is used. Both the Dirichlet and Neumann boundary conditions were addressed as source items. The generalized format is expressed as:

$$
\begin{aligned}
& a_{P} T_{P}=\sum a_{n b} T_{n b}+S_{u} \\
& a_{P}=\sum a_{n b}-S_{P}
\end{aligned}
$$

where the subscript $n b$ in the $3 \mathrm{D}$ model represents the node of $W, E, N$, $S, T$, or $B$ (see the control volume in Fig. 2). The detailed process of the discretization has been well explained in [32]. Then this series of algebraic equations was solved iteratively by the TDMA (Tridiagonal Matrix Algorithm) solver. During each calculation, the temperatures at the nodes along the South-North direction are unknown, assuming the temperature at the neighboring four nodes are known. Therefore, the calculation direction proceeds from South to North, then moves to the next line from West to East, and finally from bottom to the top until the pre-defined convergence criterion is met. Then the thermal conductivity is updated globally with the newly obtained temperature of each node. After the solution converges for the initially guessed $T_{f t p}^{k}$, the Newton-Raphson method is used to optimize the next $T_{f t p}^{k+1}$ and the TDMA solver is called again to obtain the new footprint 
temperature map.

\section{Newton-Raphson iteration}

The Newton-Raphson method for optimization is very efficient but not robust due to its dependence on the initial value. When referring to the silicon microchannel evaporator, however, a simple 1D heat conduction model can provide a sufficiently good initial guess of the footprint temperature. In the current experimental case, the temperature difference between the test section bottom and the footprint was evaluated to be about $0.4 K$ (as reported in [7], regarding the usual range of $0.15 \sim 0.8 \mathrm{~K}$ for a silicon microchannel evaporator). Therefore, the initial temperature array, $T_{f t p}^{0}$, is guessed by subtracting $0.4 \mathrm{~K}$ from the measured base temperature map $\left(T_{f t p}^{0}=T_{b a s e}-0.4\right)$. The Newton-Raphson method is described as follows:

$$
\begin{aligned}
& \delta T_{\text {base }}=T_{\text {base,cal }}-T_{\text {base,meas }} \\
& \delta T_{\text {base }_{\text {pert }}}=T_{\text {base,cal }}{ }_{\text {pert }}-T_{\text {base,meas }} \\
& T_{\text {base }_{\text {pert }}}^{\prime}=\frac{\delta T_{\text {base }_{\text {pert }}}-\delta T_{\text {base }}}{\text { pert }} \\
& T_{f t p, \text { new }}=T_{f t p, \text { old }}-\frac{\delta T_{\text {base }}}{T_{\text {base }_{\text {pert }}}^{\prime}}
\end{aligned}
$$

For each TDMA solver call (i.e. each $T_{f t p}$ map), the maximum absolute temperature difference between the two consecutive calculations was used as the convergence criterion, and was set at $10^{-9} \mathrm{~K}$ in this case. For the Newton-Raphson iteration, the convergence criteria was set as $0.05 \mathrm{~K}$ based on the maximum local difference of base temperature between the calculated and the measured one. The perturbation (pert) of the Newton-Raphson 
iteration method was chosen to be 0.0001. Normally this method yields a converged solution within two or three steps when using the properly filtered temperature map.

\section{Energy balance method}

After convergence is reached with the $3 \mathrm{D}$ heat conduction equation by the TDMA solver coupled with Newton-Raphson method noted above, the

local footprint heat flux $q_{f t p}$ is obtained by a local energy balance method for all the cells at the top layer of the grid:

$$
Q_{N}+Q_{S}+Q_{W}+Q_{E}+Q_{T}+Q_{B}=0
$$

In the above equation, $Q_{T}$ is calculated as $A_{t} q_{f t p}(y, z)$ while the other terms are obtained from the local finite volume discretized Fourier equation (taking appropriate heat transfer surfaces).

Current heat conduction models in literature

In the open literature, three heat conduction models have been described so far to reduce the experimental data in microchannel evaporators: 1DDirect, 2D-TDMA, and 3D-Direct models. These models are presented and discussed below.

\section{D-Direct model}

The popular 1D-Direct heat conduction model used in [1, 4] simply implements the Fourier law layer by layer from the base to the footprint, as shown in Fig. 4a. It is referred as a "direct" model since it uses the 2 boundary conditions at the base layer (i.e. the temperature and the heat flux) without 
iterating on the boundary condition at the footprint. The boundary conditions on all lateral sides of the substrate are considered to be adiabatic and the governing equation of $1 \mathrm{D}$ heat conduction model is thus:

$$
q=-k \frac{\partial T}{\partial x}, T(z)=T_{I R}(z), q(z)=q_{b}(z)
$$

Considering that the thermal conductivity of silicon is temperature dependent, its relation with temperature is accounted for as:

$$
k=0.0007 T^{2}-0.5416 T+157.39
$$

In the 1D-Direct model, the discretization is only performed in the thickness direction $x$, and the proper number of layers in the grid is identified through a grid independent analysis. The number of calculation points along the evaporator lengthwise, i.e. along the flow direction, is determined by the number of temperature measurement points.

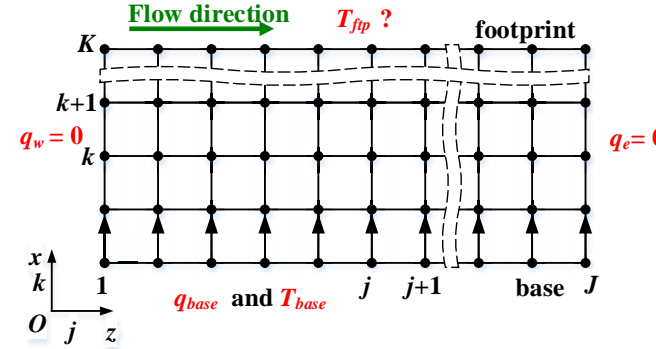

(a) Mesh in 1D model

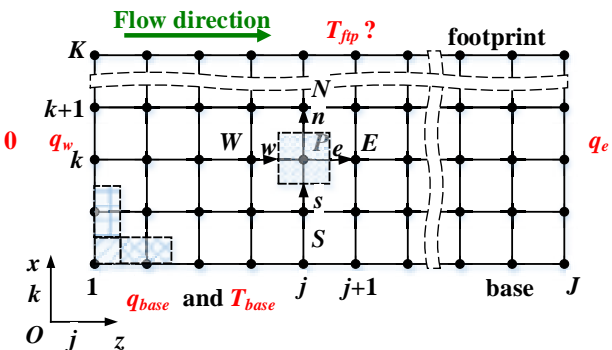

(b) Mesh in 2D model

Fig. 4: Mesh types used in 1D-Direct and 2D-TDMA models. 


\section{D-TDMA model}

The 2D heat conduction model deals with both thickness and lengthwise heat transfer, i.e. the $x z$ plane in Fig. $4 \mathrm{~b}$. The steady state $2 \mathrm{D}$ heat conduction equations and its boundary conditions are expressed as follows:

$$
\begin{aligned}
& \frac{\partial}{\partial x}\left(k \frac{\partial T}{\partial x}\right)+\frac{\partial}{\partial z}\left(k \frac{\partial T}{\partial z}\right)=0 \\
& T(0, z)=T_{I R}(z), k \frac{\partial T}{\partial x}(0, z)=q_{b}(z) \\
& k \frac{\partial T}{\partial z}(x, 0)=q_{w}(z), k \frac{\partial T}{\partial z}\left(x, L_{M E}\right)=q_{e}(x)
\end{aligned}
$$

Contrary to the 1D-Direct model, the 2D-TDMA model uses only one boundary condition (i.e. temperature or heat flux) at the base and iterates on the footprint boundary condition. In [25] the 2D heat conduction model was solved iteratively based on the finite volume method by a TDMA solver, and a guessed Robin boundary condition at the footprint. However, this boundary condition, as mentioned previously, is time-expensive to obtain a converged solution. Therefore, this Robin condition has been modified here to evaluate this approach by guessing a Dirichlet boundary condition at the footprint. The discretized 2D heat equation is similar to Eq. (19) and Eq. (20), while the subscript $n b$ in 2D model represents $W, E, N$, or S. Similar to the 3D-TDMA model, the 2D-TDMA model is solved by a TDMA solver based on the finite volume method combining the Newton-Raphson iteration. The footprint heat flux is then obtained through the local energy balance method. 


\section{D-Direct model}

The 3D direct heat conduction model is derived from the energy balance method, which is the sum of the net heat entering the central node from three dimensions being equal to zero in steady state. The 3D heat equation is solved directly from the bottom of the test section to the footprint, in the same manner as the 1D-Direct model (i.e. without iteration). For the internal nodes, the discretized equations are as follows:

$$
\begin{aligned}
& Q_{N}+Q_{S}+Q_{W}+Q_{E}+Q_{T}+Q_{B}=0 \\
& Q_{B}=A_{b} k_{b}[T(i, j, k-1)-T(i, j, k)] / \Delta x \\
& Q_{T}=A_{t} k_{t}[T(i, j, k+1)-T(i, j, k)] / \Delta x \\
& Q_{N}=A_{n} k_{n}[T(i+1, j, k)-T(i, j, k)] / \Delta y \\
& Q_{S}=A_{s} k_{s}[T(i-1, j, k)-T(i, j, k)] / \Delta y \\
& Q_{W}=A_{w} k_{w}[T(i, j-1, k)-T(i, j, k)] / \Delta z \\
& Q_{E}=A_{e} k_{e}[T(i, j+1, k)-T(i, j, k)] / \Delta z
\end{aligned}
$$

For the peripheral nodes, their $Q$ and $A$ vary depending on the local boundary condition. For example, when this method is employed at the first layer (the test section bottom), the temperature of the upper layer can be obtained with the known $Q_{B}=A_{b} q_{b}$ and $T_{b a s e}$. Additionally, the thermal conductivity is updated with the new current temperature value. Then, progressively using the energy balance method from bottom to the top, the local temperature and heat flux at the footprint are obtained. Again, a grid independent analysis is used to determine the proper number of layers along the thickness direction, while the IR pixel spacings are used in the $y z$ planar directions. This method 
has been used in the work of [8].

\section{Analysis of results and discussion}

\section{Filtering techniques comparison}

In the present section, the 3D IHCP was solved with the pre-processed temperature from the three filtering techniques previously described. The results based on the two-phase experimental data are compared in order to decide which of these techniques is better to be adopted.

Prior to the comparison of the filtering methods, a grid independence analysis was performed along the $x$-direction, i.e. across the thickness of the substrate. The number of grid cells in the $y z$ plane (as denoted in Fig. 2) are fixed according to the IR camera pixel numbers, which are $120 \times 120$ in this case and uniformly distributed. The number of grid cells in the $x$-direction was successively set to 5, 10 and 20 and the 3D IHCP was solved using the filtered data obtained from the polynomial surface fitting. The maximum absolute variation in $q_{f t p}$ from 10 to 20 grid cells was only $0.2 \%$ while the computational time was increased to $2627 \mathrm{~s}$ from $271.9 \mathrm{~s}$ (a factor of 10). Hence the grid type of $10 \times 120 \times 120$ was chosen and used for all the results further presented.

The Wiener filter is applied twice as suggested in [16] to remove the noise and then to smooth the data. The smoothing factor used in the spline smoothing function is 8. In polynomial surface fitting, the orders of 4 and 4 are chosen respectively for the fits in the axial and perpendicular flow directions based on the filtering precision evaluated by Eq. (38). The least absolute residual method is used as the robust linear least-squares fitting method. 
Fig. 5 describes the filtering details with the three techniques against the raw IR temperature data of the 70th row $(i=70)$ along the flow direction. It is shown that (1) the spline smoothing technique generated the closest results to the raw data, (2) the polynomial surface fitting gave the purely smooth results with relatively higher error as a compromise, and (3) the Wiener filter yielded only moderate results. The mean of the temperature difference square between the filtered and measured ones is used to evaluate the performance of a filtering approach. This value is defined as:

$$
\left.\xi=\frac{1}{E \cdot F} \sum_{i, j \in O}\left[T_{e x p}(i, j)-\tilde{T}_{e x p}(i, j)\right)\right]^{2}
$$

When the mean of this value is below or close to the standard deviation of the raw temperature data [16], which is $0.07 \mathrm{~K}$ according to the present set from the IR camera, the filtered temperature is considered to be sufficiently accurate to be used for solving the inverse problem. Table 1 lists the difference between the three filtering approach in terms of error $\xi$, Newton-Raphson iteration steps and computation time to solve the entire 3D IHCP. Since each $\xi$ of the three filtering techniques is found to be less than $0.07 \mathrm{~K}$, all of them provide a sufficiently accurate temperature map for solving the IHCP.

Based on the three temperature filtering techniques, the footprint heat flux $q_{f t p}$ was obtained by solving the 3D IHCP and the results are shown in Fig. 6. It is found that the results based on the Wiener filter oscillated immensely, although the filtered IR temperatures appeared to be relatively smooth (see Fig. 5). This is because that the high-resolution temperature map $(120 \times 120$ pixels in a small area of $\left.0.01 \times 0.01 \mathrm{~m}^{2}\right)$ causes an oscillation in the second 


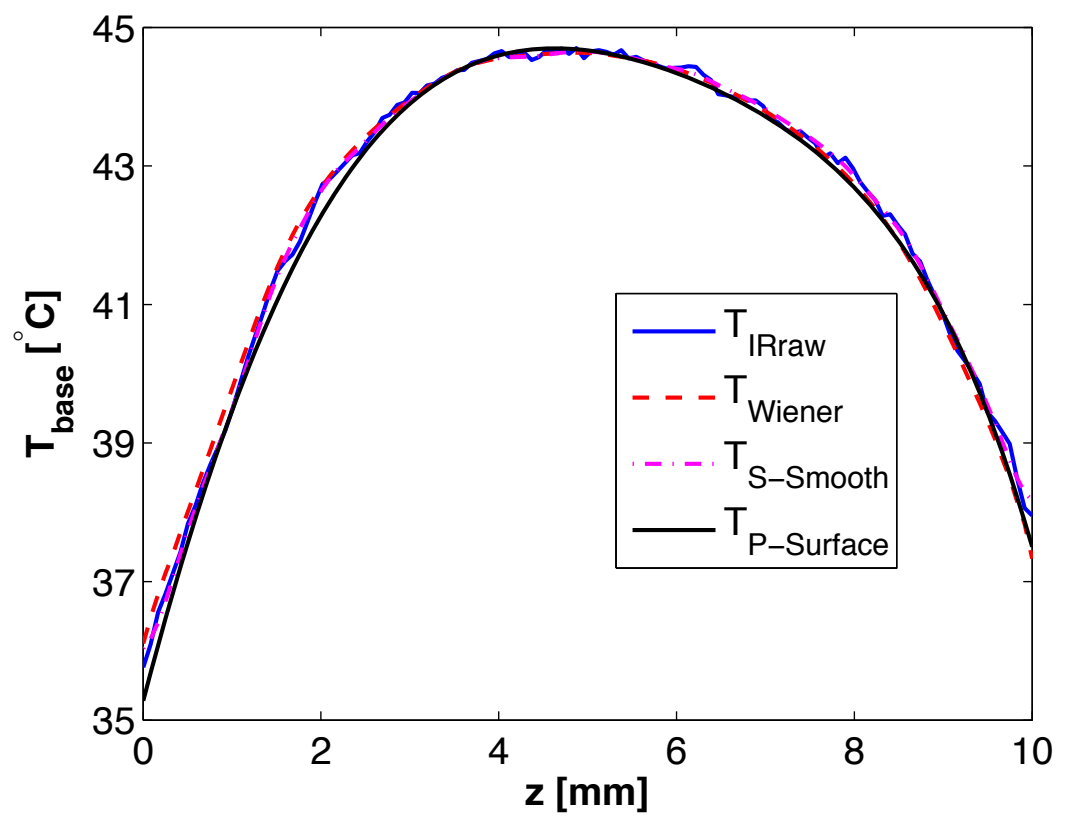

Fig. 5: Filtering results comparison against raw IR temperature $(i=70)$.

derivative of the filtered temperature if the filter method is not appropriate. The results from the spline smoothing function fluctuated moderately around that from the polynomial surface fitting, though its corresponding $\xi$ shown in Table 2 was the smallest one. This means the two filters are not robust enough to remove the impact of the signal noise on the Laplacian item, although they generated numerically converged solutions. On the other hand,

Table 2: Results from the three temperature filtering techniques.

\begin{tabular}{lccc}
\hline Technique & $\xi$ & NRsteps & Time(s) \\
\hline Wiener filter & 0.0403 & 317 & 6201.2 \\
Spline smooth & 0.0066 & 6 & 118.4 \\
Polynomial surface & 0.0601 & 2 & 271.9 \\
\hline
\end{tabular}


the polynomial surface fitting generated smooth results with a reasonable calculation time.

In order to retain the original spatial fine resolution of temperature measure-

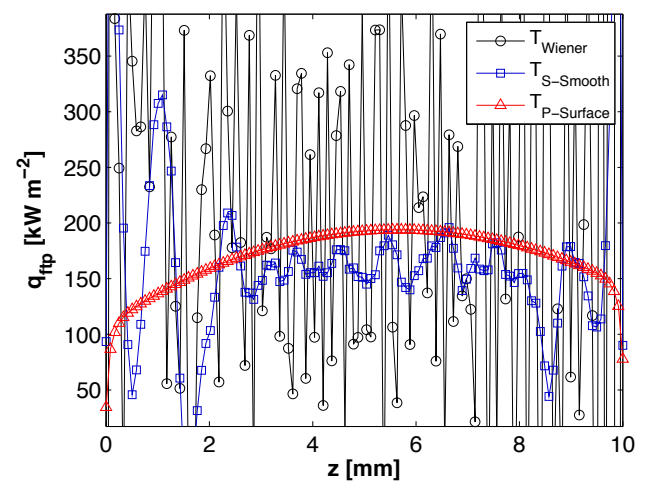

Fig. 6: Comparison of two-phase flow widthwise averaged local footprint heat flux from the three temperature filtering techniques.

ments $\left(120 \times 120\right.$ pixels in an area of $\left.0.01 \times 0.01 \mathrm{~m}^{2}\right)$, for our application, the success of solving the inverse heat conduction problem here to reduce the local heat transfer data comes from both the low noise level in our data and the smoothness of the data after filtering. As shown in Fig. 5, the Wiener filter and the spline smoothing function has generated nearly noise-free but not continuously differentiable data (ignoring the discontinuity of data), which are required by the present fine spatial resolution when calculating the second derivative item in heat equations in order to reach a physical solution of the footprint heat flux. This is also the reason why significantly non-physical oscillations in footprint heat flux obtained from the filtered data by the forgoing two methods were found in Fig. 6.

Therefore, the polynomial surface fitting technique is recommended to filter the raw IR temperature and has been employed for the experimental data 
reduction. Additionally, it is worthwhile to mention that this polynomial surface fitting technique is suitable for high-resolution temperatures, but is not appropriate for the case with a point hot spot, since the point information will be smoothed out.

\section{Comparison of heat conduction models}

Based on the filtered temperatures obtained using the polynomial surface fitting technique, $q_{f t p}$ and $T_{f t p}$ were obtained through the various heat conduction models described previously. In order to compare with one dimensional values of $q_{f t p}$ and $T_{f t p}$ from the 1D-Direct and 2D-TDMA models, the results from 3D-Direct and 3D-TDMA models were averaged along the widthwise direction.

Case I: Single-phase flow

Footprint heat flux and temperature. Fig. 7a shows the different local footprint heat fluxes based on the four heat conduction models (i.e. the three found in the open literature and the new one). The first and last 20 original grid cells are kept to maintain the original trend, while in the middle a sparse grid is presented for a clearer view. The 3D-Direct model introduces a non-physical oscillation at the regions close to the boundary. The extreme values were cut from the current plot to maintain a good viewing scale. The footprint heat flux from the 3D-Direct model in most of the regions are lower than those from the rest of the models. This discrepancy comes from the considerable amount of heat conducted to the ends when considering the total energy balance. However, the TDMA models (2D and 3D) generate reasonable results, which are close to the results from the 1D-Direct model. 
Fig. $7 \mathrm{~b}$ describes the trend of local footprint temperature along the flow

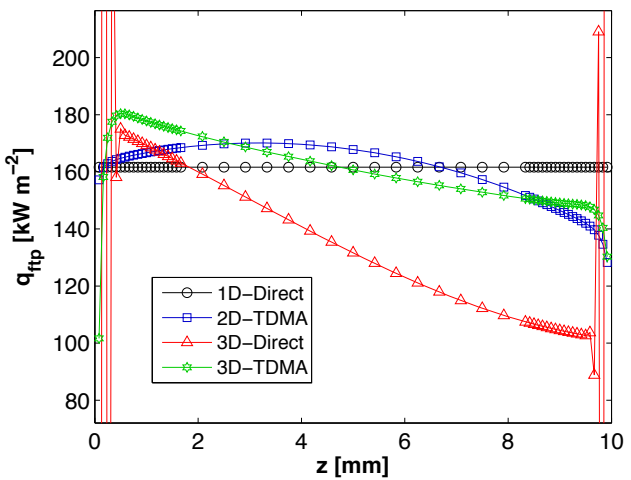

(a) Single-phase $q_{f t p}$

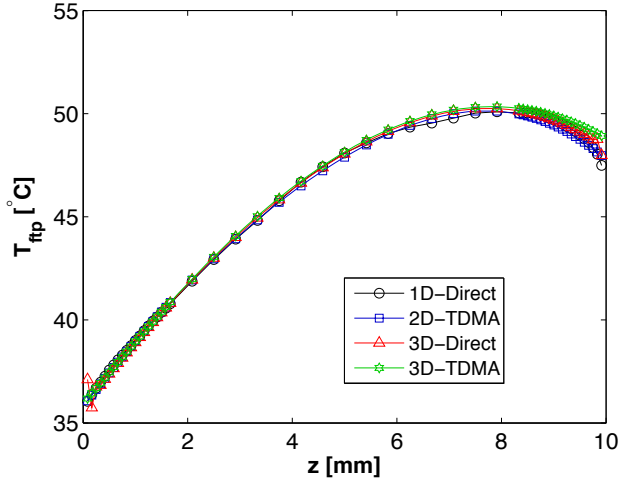

(b) Single-phase $T_{f t p}$

Fig. 7: Single-phase flow local footprint heat flux and temperature (sparse grid in the middle is used for a clearer view).

direction. It is shown that the footprint temperature first increases nearly linearly versus length, then decreases when approaching the channel outlet. This decreasing trend might be due to the heat spreading from the test section to the manifold. From the results of 3D-TMDA model, the maximum temperature difference between the base and the footprint was $0.4 \mathrm{~K}$.

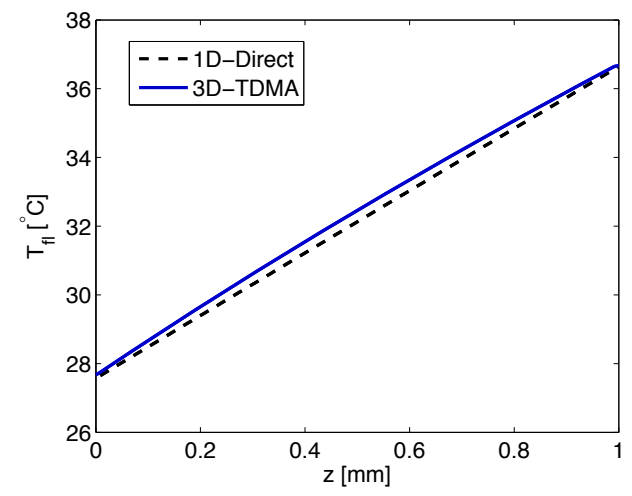

Fig. 8: Single-phase flow fluid temperature along the flow direction. 
Local fluid temperature, heat transfer coefficients, and Nusselt number. Following the analysis presented in [33], the axial heat conduction in the fluid needs to be taken into account in the regions where $z \leq 10 \frac{D_{h}}{P e}$. For the current single-phase flow case at $\mathrm{G}=2000 \mathrm{~kg} \mathrm{~m}^{-2} \mathrm{~s}^{-1}$, the corresponding length $z$ is $0.325 \mu \mathrm{m}$, which is negligible. The fluid temperature in single-phase flow is usually assumed to increase linearly along the flow direction based on the premise of a uniform heat flux imposed on the test section backside [1]. However, one might note that the $q_{f t p}$ as discussed previously is not uniform even though the base heat flux is constant. Thus the fluid temperature is unlikely to increase linearly. Fig. 8 represents the local fluid temperature based on the 1D-Direct model and 3D-TDMA model. The 1D-Direct model predicted a linear rise of the fluid temperature. However, the result from the 3D-TDMA model revealed that the fluid temperature increased with a parabolic trend but shared the same outlet temperature with that from the 1D model due to the total energy balance. The maximum difference in fluid temperature between the two models is $0.3 \mathrm{~K}$.

The local wall and footprint heat transfer coefficients and the fin efficiency based on the 3D TDMA heat conduction model are plotted in Fig. 9. The heat transfer coefficients between the wall and the footprint are linked by the fin efficiency, which is very close to 1 in the current experimental case. For this experimental condition, the thermal entrance length, $L_{t h}=0.05 \operatorname{Re} D_{h} \operatorname{Pr}=0.0154 \mathrm{~m}$, is longer than the channel length of 0.01 m. Therefore, the fluid is always thermally developing. In such a case, it is known that the heat transfer coefficients continuously decreases along the channel. In our case, the slight increase towards the channel exit is consid- 


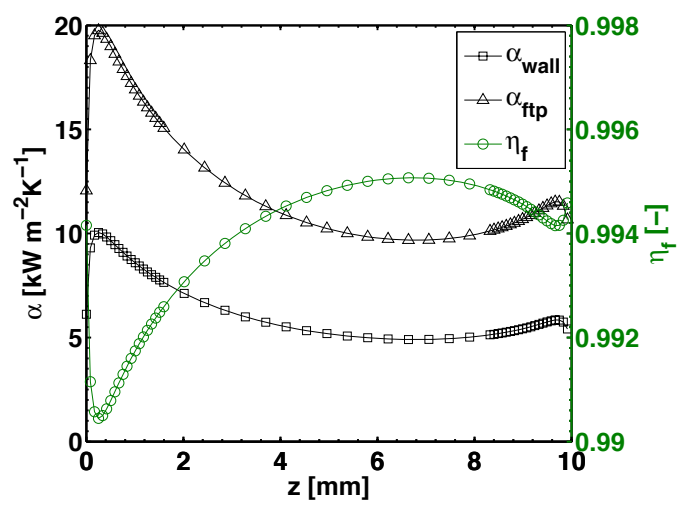

Fig. 9: Single-phase flow local heat transfer coefficients and fin efficiency obtained with the new 3D-TDMA model.

ered to be related to the heat spreading to the manifold.

Fig. 10 compares the predicted local Nusselt number from the correlation

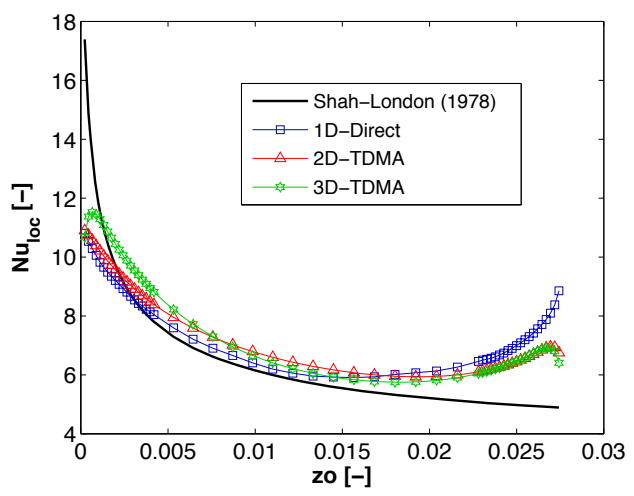

Fig. 10: Single-phase flow local Nusselt number comparison.

of Shah and London [34] with the experimental ones based on the 1D-Direct, 2D-TDMA and 3D-TDMA models, where $z o$ is a dimensionless number defined as $z o=\frac{\pi z}{4 \operatorname{Re} \operatorname{Pr} D_{h}}$. The agreement between prediction and the experimental results is good except for areas close to the channel exit, where the experimental value is higher than the predicted one. This is mainly due to the 
heat conducted to the manifold. To further evaluate the performance of the three heat conduction models, the mean absolute errors (MAE) were obtained to be $14.47 \%, 13.08 \%$ and $11.81 \%$ respectively for 1D-Direct, 2D-TDMA and 3D-TDMA models. Note that the values near the inlet are influenced by the inlet orifice. Therefore, the 3D-TDMA model is proven to be more accurate for reducing the experimental data of such a multi-microchannel evaporator.

Case II: Two-phase flow

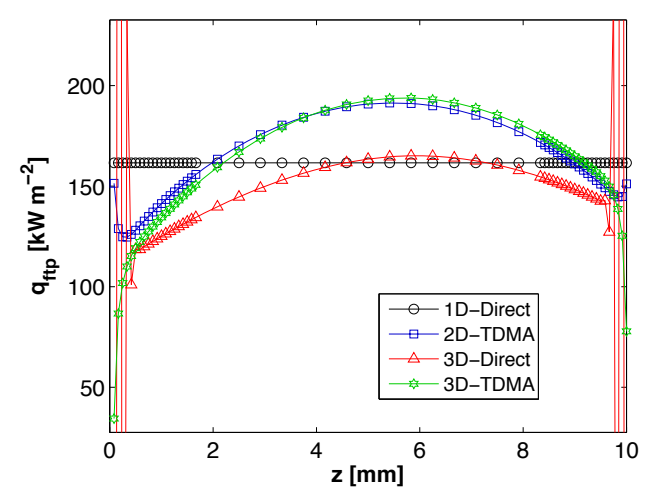

(a) Two-phase $q_{f t p}$

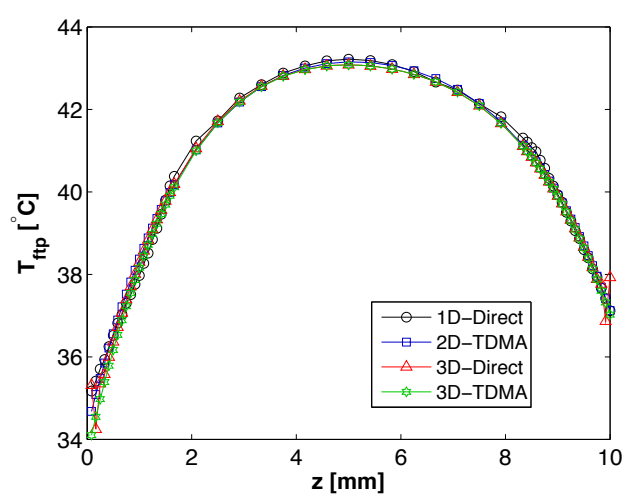

(b) Two-phase $T_{f t p}$

Fig. 11: Two-phase flow local footprint heat flux and temperature.

Footprint heat flux and temperature. Fig. 11 shows the local $q_{f t p}$ and $T_{f t p}$ at the footprint of the two-phase flow test based on the four different models. The 1D-Direct model simply predicted a constant value, which is as expected the same as $q_{\text {base }}$. The 2D-TDMA and 3D-TDMA models yielded similar results especially in the middle region. In the 2D-TDMA model, the unrealistic slight rise of heat flux at areas close to the two endings is resulted from ignoring the heat conduction in widthwise direction. The $q_{f t p}$ from the 3D-Direct model strongly oscillated from -2.83 to $2.14 \times 10^{3} \mathrm{~kW} \mathrm{~m}^{-2}$ in 
the regions close to the boundary (extreme values were cut from the current plot to make a suitable scale). However, these non-physical fluctuations (already observed in the single-phase results) are not present in both the 2DTDMA and 3D-TDMA models, which predicted the results smoothly in the entire region. Comparing the 3D-Direct model with the 2D- and 3D-TDMA models, these oscillations at regions close to the boundary are related to the manner of solving the heat conduction equations. As discussed previously, the $2 \mathrm{D}$ or $3 \mathrm{D}$ heat conduction model is a non-linear elliptical equation. This requires a closed set of boundary conditions to be simultaneously involved in the calculation process. However, one might notice that in the direct model the calculation proceeds from the bottom to the top without any iteration, using the boundary conditions at the bottom and lateral sides without the one at the top. In this case, the errors due to the less precise estimation of the boundary condition at the lateral sides are magnified during the calculation process. On the other hand, the 2D- or 3D-TDMA model is solved iteratively with closed boundary conditions. It assumes a Dirichlet boundary condition at the top and uses the measured Dirichlet one (base temperature from IR camera) as the investigator (i.e. to correct the footprint temperatures upon Newton-Raphson iterations). Hence, the results from the 3D-TDMA model are smooth and realistic. ,

Additionally, the 3D-TDMA model provides high-resolution thermal maps for all variables of interest. Fig. 12 compares $q_{f t p}$ and $T_{f t p}$ between singleand two-phase flow sharing the same heat flux and mass flux but different inlet and saturation temperatures. To reduce the impact of heat spreading on the scales, five pixels at each lateral side were cut off. It is shown that 
the $q_{f t p}$ of two-phase flow was higher than that of single-phase flow, while the two-phase $T_{f t p}$ was lower. For example, the maximum $T_{f t p}$ of two-phase flow is $44.8^{\circ} \mathrm{C}$, whereas that of single-phase flow is $51.9^{\circ} \mathrm{C}$, considering the inlet fluid temperatures for the single- and two-phase flow were respectively 27.4 and $30.7^{\circ} \mathrm{C}$. The $2 \mathrm{D}$ thermal map demonstrates that the two-phase flow renders the local heat flux and temperature distribution more uniform. Therefore, it is clearly shown that the thermal performance of the two-phase flow exceeds that of single-phase flow.

Fig. 13 presents the two-phase local wall and footprint heat transfer coefficients and the local fin efficiency at the widthwise centerline along the flow direction. According to the local vapor quality evaluated from the local energy balance method, the flow along the channel was separated into two region: the single-phase or subcooled flow boiling region and the two-phase saturated flow boiling region. The fluid temperature in the former region was calculated based on the single-phase energy balance, while the fluid temperature in the saturated flow boiling region was evaluated based on the local fluid pressure, which is normally predicted by an appropriate empirical correlation. However, a linear two-phase flow pressure drop assumption was made here due to its simplicity to obtain the local fluid temperature. It is shown that the local wall heat transfer coefficient first decreased in the single-phase thermal developing region, then it increased in the subcooled flow boiling region to a peak which is the onset of saturated flow boiling; afterwards, it experienced a very slight decrease and finally picked up after entered into annular flow regime. Therefore, the variation of two-phase local heat transfer coefficients in big magnitude emphasizes the necessity of considering the 


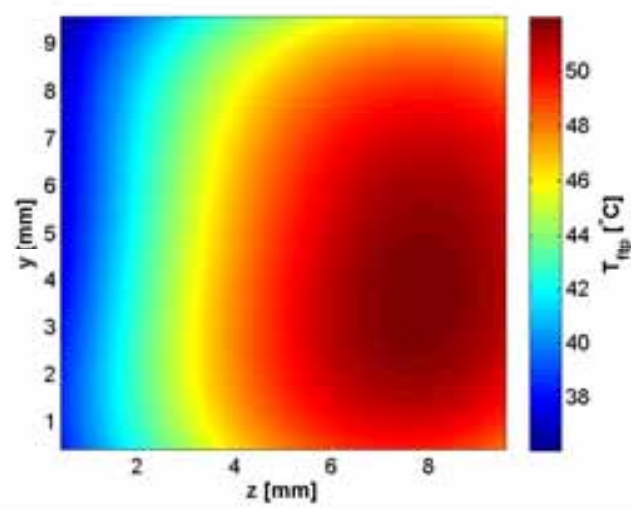

(a) Single-phase $T_{f t p}$

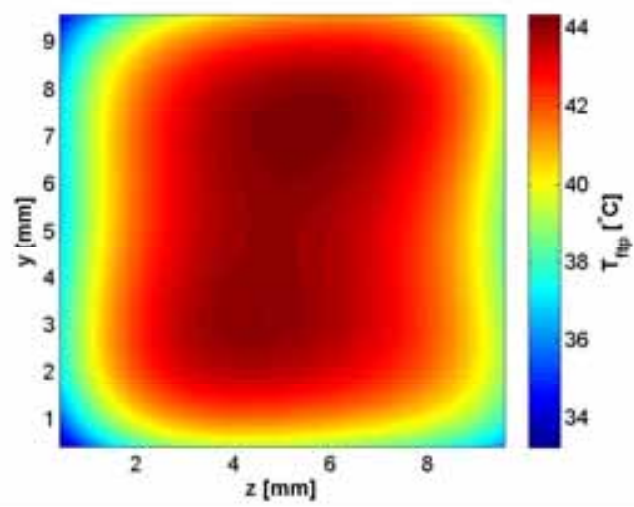

(c) Two-phase $T_{f t p}$

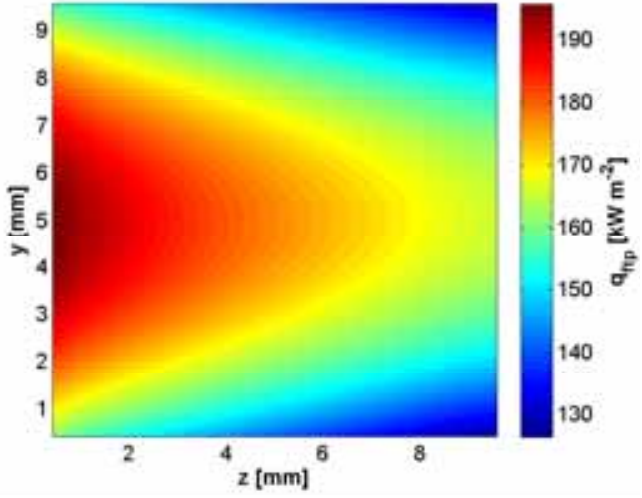

(b) Single-phase $q_{f t p}$

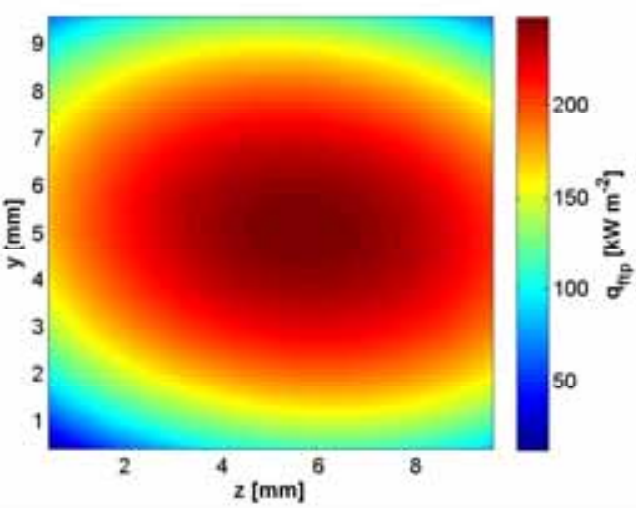

(d) Two-phase $q_{f t p}$

Fig. 12: Single- and two-phase flow thermal maps of footprint heat flux and temperature.

non-uniform distribution of the local footprint heat flux.

\section{Conclusion}

The problem of determining the local convective heat transfer coefficients at the interface between the fluid and channel wall in multi-microchannel evaporators using temperature measurements at the base is characterized as an inverse heat conduction problem (IHCP). In this paper, a new method of 


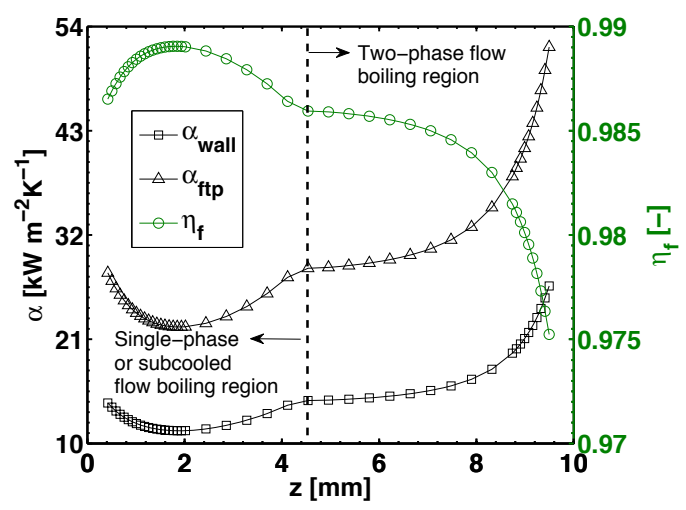

Fig. 13: Two-phase flow local heat transfer coefficients and fin efficiency at the widthwise centerline, obtained from the new 3D-TDMA model.

reducing the local heat transfer data in multi-microchannel evaporators by solving the 3D IHCP has been proposed. To validate this method, two sets of single- and two-phase experimental data in a multi-microchannel evaporator were used. In the experiment, the base temperature map was measured by an infrared camera, providing not only high-resolution temperature information but also a good reference to define the lateral boundary conditions. In this new method, the raw temperature map were first pre-processed by filtering techniques, including Wiener filter, spline smoothing, and polynomial surface fitting. Then the 3D IHCP was solved using the TDMA finite volume method with a combination of the Newton-Raphon and local energy balance methods. The result indicated that the polynomial surface fitting technique was more suitable to be adopted in the new method to process the present experimental data.

Based on the filtered data from surface fitting, three other heat conduction models currently used in the literature, including 1D-Direct, 2D-TDMA, and 
3D-Direct models, were systemically analyzed. The results were compared with that from the 3D-TDMA model used in the present new method. The results of local footprint heat flux $q_{f t p}$ and temperature $T_{f t p}$ showed that the new method presented more reasonable solutions, while the direct models gave irrational oscillations in the boundary regions. These non-physical fluctuation were essentially related to the progressively magnified error, which arose from the not-so-precise estimation of the Neumann boundary condition at the lateral sides.

Furthermore, the single-phase flow local Nusselt number results indicated that the new method provided the best agreement with the theoretical prediction with a MAE of $11.8 \%$. Finally, the two-phase flow was proven to exhibit higher $q_{f t p}$ and lower temperature $T_{f t p}$ than single-phase flow, and the distributions appeared to be more uniform than those for the single-phase flow. The latter result highlights the efficiency of this 3D-TDMA model to obtain proper physical solutions for the problem of heat conduction in the frame of experimental microchannel evaporator measurements.

As a conclusion, the present 3D-TDMA method of solving the 3D IHCP using polynomial surface fitting, the 3D-TDMA model, Newton-Raphson iteration and a local energy balance method was shown to be more accurate for reducing the local heat transfer data in multi-microchannel evaporators, especially for cases using IR measurements, since such flows exhibit very large and sharp variations in the local heat transfer coefficient as a function of two-phase flow regime transitions. Extensive two-phase heat transfer coefficient data will be obtained using this new method and will be presented in future papers. 


\section{Nomenclature}

\begin{tabular}{|c|c|}
\hline Roman & Description \\
\hline$A$ & cross section area $\left(\mathrm{mm}^{2}\right)$ \\
\hline$C$ & coefficients $(-)$ \\
\hline$c_{p}$ & heat capacity $\left(\mathrm{J} \mathrm{kg}^{-1} \mathrm{~K}^{-1}\right)$ \\
\hline$D_{h}$ & hydraulic diameter (m) \\
\hline$E$ & pixel numbers \\
\hline Er & mean square temperature difference $(\mathrm{K})$ \\
\hline$F$ & pixel numbers \\
\hline$G$ & mass flux $\left(\mathrm{kg} \mathrm{m}^{-2} \mathrm{~s}^{-1}\right)$ \\
\hline$I$ & grids number in widthwise (-) \\
\hline$i$ & grid index in widthwise $(-)$ \\
\hline$J$ & grids number in lengthwise (-) \\
\hline$j$ & grid index in lengthwise (-) \\
\hline$K$ & grids number in thickness direction (-) \\
\hline$k$ & thermal conductivity $\left(\mathrm{W} \mathrm{m} \mathrm{m}^{-1} \mathrm{~K}^{-1}\right)$ \\
\hline$L$ & length $(\mathrm{m})$ \\
\hline$m$ & fin parameter $\left(\mathrm{m}^{-1}\right)$ \\
\hline$\dot{m}$ & mass flux $\left(\mathrm{kg} \mathrm{m}^{-2} \mathrm{~s}^{-1}\right)$ \\
\hline$N$ & microchannel number (-) \\
\hline$N A$ & noise amplitude $(\mathrm{K})$ \\
\hline$N u$ & Nusselt number (-) \\
\hline$P e$ & Peclet number (-), $P e=R e P r$ \\
\hline $\operatorname{Pr}$ & Prandtl number (-), $\operatorname{Pr}=\frac{c_{p} \mu}{k}$ \\
\hline pert & protuberance $\left({ }^{\circ} \mathrm{C}\right)$ \\
\hline
\end{tabular}




$\begin{array}{ll}Q & \text { heat transfer rate }(\mathrm{W}) \\ q & \text { heat flux }\left(W \mathrm{~m}^{-2}\right) \\ R & \text { temperature difference }\left({ }^{\circ} \mathrm{C}\right) \\ R e & \text { Reynolds number }(-), R e=\frac{G D_{h}}{\mu} \\ S & \text { smoothing factor }(-) \\ T & \text { temperature }(\mathrm{K}) \\ \tilde{T} & \text { filtered temperature, }(\mathrm{K}) \\ v & \text { noise variance }(-) \\ W & \text { width (m) } \\ x & \text { dimension in thickness direction }(\mathrm{m}) \\ y & \text { dimension in span wise }(\mathrm{m}) \\ z & \text { dimension in length wise }(\mathrm{m})\end{array}$

\section{Greek}

$\begin{array}{ll}\alpha & \text { heat transfer coefficient }\left(\mathrm{W} \mathrm{m}^{-2} \mathrm{~K}^{-1}\right) \\ \Delta & \text { difference }(-) \\ \mu & \text { mean value }(-) \\ \Omega & \text { local neighborhood }(-) \\ \sigma & \text { variance }(-) \\ \xi & \text { mean of temperature difference square }(-) \\ \text { Subscripts } & \\ \text { axial } & \text { axial heat conduction } \\ b & \text { bottom } \\ \text { base } & \text { test section base } \\ \text { cal } & \text { calculated } \\ \text { exp } & \text { experiment }\end{array}$




$\begin{array}{ll}\text { ch } & \text { channel } \\ e & \text { east } \\ f & \text { fin } \\ f t p & \text { footprint } \\ i & \text { index } \\ j & \text { index } \\ \text { loss } & \text { heat loss } \\ M E & \text { microchannel evaporator } \\ n & \text { north } \\ n o & \text { noise } \\ s & \text { south } \\ t & \text { top } \\ \text { total } & \text { total } \\ w & \text { west } \\ w a l l & \text { channel wall }\end{array}$




\section{Acknowledgment}

The China Scholarship Council was acknowledged for part of financial support to H. Huang. N. Lamaison was supported by the Swiss National Science Foundation (SNSF) and sponsored by Nano-Tera project YINS.

\section{References}

[1] W. Qu, I. Mudawar, Experimental and numerical study of pressure drop and heat transfer in a single-phase micro-channel heat sink, Int. J. Heat Mass Transf. 45 (12) (2002) 2549-2565.

[2] E. Costa-Patry, J. R. Thome, On-chip cooling of hot-spots with a copper micro-evaporator, Annu. IEEE Semicond. Therm. Meas. Manag. Symp. (2012) 125-129.

[3] G. Hetsroni, A. Mosyak, Z. Segal, G. Ziskind, A uniform temperature heat sink for cooling of electronic devices, Int. J. Heat Mass Transf. 45 (16) (2002) 3275-3286.

[4] J. Xu, Y. Gan, D. Zhang, X. Li, Microscale heat transfer enhancement using thermal boundary layer redeveloping concept, Int. J. Heat Mass Transf. 48 (9) (2005) 1662-1674.

[5] D. Krebs, V. Narayanan, J. Liburdy, D. Pence, Spatially resolved wall temperature measurements during flow boiling in microchannels, Exp. Therm. Fluid Sci. 34 (4) (2010) 434-445.

[6] G. M. Carlomagno, G. Cardone, Infrared thermography for convective heat transfer measurements, Exp. Fluids 49 (6) (2010) 1187-1218. 
[7] G. Hetsroni, A. Mosyak, E. Pogrebnyak, R. Rozenblit, Infrared temperature measurements in micro-channels and micro-fluid systems, Int. J. Therm. Sci. 50 (6) (2011) 853-868.

[8] S. Szczukiewicz, N. Borhani, J. R. Thome, Fine-resolution two-phase flow heat transfer coefficient measurements of refrigerants in multimicrochannel evaporators, Int. J. Heat Mass Transf. 67 (2013) 913-929.

[9] J. Beck, B. Blackwell, C. S. Clair, Inverse Heat Conduction Problem: Ill Posed Problems, Wiley-Interscience: New York, 1985.

[10] A. Tikhonov, V. Arsenin, Solution of Ill Posed Problems, Winston-Sons, Washignton, DC, 1977 .

[11] O. Alifanov, Inverse Heat Transfer Problems, Springer-Verlag, New York, 1994 .

[12] H. R. Orlande, O. Fudym, D. Maillet, R. M. Cotta, Thermal Measurements and Inverse Techniques, 2011.

[13] Y. Rouizi, D. Maillet, Y. Jannot, Fluid temperature distribution inside a flat mini-channel: Semi-analytical wall transfer functions and estimation from temperatures of external faces, Int. J. Heat Mass Transf. 64 (2013) $331-342$.

[14] Y. Rouizi, W. A. Hadad, D. Maillet, Y. Jannot, Experimental assessment of the fluid bulk temperature profile in a mini channel through inversion of external surface temperature measurements, Int. J. Heat Mass Transf. 83 (2015) 522-535. 
[15] S. Rainieri, G. Pagliarini, Data filtering applied to infrared thermographic measurements intended for the estimation of local heat transfer coefficient, Exp. Therm. Fluid Sci. 26 (2-4) (2002) 109-114.

[16] F. Bozzoli, G. Pagliarini, S. Rainieri, Experimental validation of the filtering technique approach applied to the restoration of the heat source field, Exp. Therm. Fluid Sci. 44 (2013) 858-867.

[17] D. Delpueyo, X. Balandraud, M. Grédiac, Heat source reconstruction from noisy temperature fields using an optimised derivative Gaussian filter, Infrared Phys. Technol. 60 (2013) 312-322.

[18] M. Ozisik, Heat Conduction - 2nd ed., Wiley-Interscience, 1993.

[19] F. Bozzoli, S. Rainieri, Comparative application of CGM and Wiener filtering techniques for the estimation of heat flux distribution, Inverse Probl. Sci. Eng. 19 (4) (2011) 551-573.

[20] M. Piasecka, S. Hozejowska, M. E. Poniewski, Experimental evaluation of flow boiling incipience of subcooled fluid in a narrow channel, Int. J. Heat Fluid Flow 25 (2) (2004) 159-172.

[21] S. Hozejowska, M. Piasecka, M. E. Poniewski, Boiling heat transfer in vertical minichannels. Liquid crystal experiments and numerical investigations, Int. J. Therm. Sci. 48 (6) (2009) 1049-1059.

[22] S. Luciani, D. Brutin, C. Le Niliot, O. Rahli, L. Tadrist, Flow boiling in minichannels under normal, hyper-, and microgravity: local heat transfer analysis using inverse methods, J. Heat Transfer 130 (10) (2008) 101502. 
[23] S. N. Ritchey, J. A. Weibel, S. V. Garimella, Local measurement of flow boiling heat transfer in an array of non-uniformly heated microchannels, Int. J. Heat Mass Transf. 71 (2014) 206-216.

[24] N. Lamaison, J. Braz Marcinichen, J. Richard Thome, Two-phase flow control of electronics cooling with pseudo-CPUs in parallel flow circuits: dynamic modeling and experimental evaluation, J. Electron. Packag. 135 (3) (2013) 030908.

[25] N. Lamaison, Dynamic modeling and experimental evaluation of a controlled two-phase on-chip cooling system designed for high efficiency datacenter, Ph.D thesis, 2014 .

[26] S. J. Kline, F. A. McClintock, Describing uncertainties in single-sample experiments, ASME Mechanical Engineering 75 (1) (1953) 3-8.

[27] C. Teodosiu, G. Rusaouën, R. Hohotă, Influence of boundary codition$\mathrm{s}$ uncertainties on the simulation of ventilated encolsures., journal $=$ Numerical Heat Transfer, pages $=483-504$, volume $=44$, year $=2003$ (January).

[28] S. Rainieri, F. Bozzoli, G. Pagliarini, Wiener filtering technique applied to thermographic data reduction intended for the estimation of plate fins performance, Exp. Therm. Fluid Sci. 28 (2-3) (2004) 179-183.

[29] P. Craven, G. Wahba, Smoothing noisy data with spline functions, Numerische Mathematik (3) (1979) 377-403.

[30] T. El Abbass, C. Jallouli, Y. Albouy, M. Diament, A comparison of 
surface fitting algorithms for geophysical data, Terra Nov. 2 (5) (1990) 467-475.

[31] P. Dierckx, An algorithm for surface-fitting with spline functions, IMA J Numer Anal 3 (1) (1981) 267-283.

[32] S. Patankar, Numerical Heat Transfer and Fluid Flow, McGraw-Hill, New York, 1980 .

[33] G. Hetsroni, A. Mosyak, E. Pogrebnyak, L. Yarin, Heat transfer in micro-channels: Comparison of experiments with theory and numerical results, Int. J. Heat Mass Transf. 48 (25-26) (2005) 5580-5601.

[34] R. Shah, A. London, Laminar Flow Forced Convection in Ducts, Academic Press, New York, 1978. 